\title{
Acceleration of cosmic rays by young core-collapse supernova remnants
}

\author{
I. Telezhinsky ${ }^{1,2}$, V. V. Dwarkadas ${ }^{3}$, and M. Pohl ${ }^{1,2}$
}

\author{
1 DESY, Platanenallee 6, 15738 Zeuthen, Germany \\ e-mail: igor.telezhinsky@desy.de \\ 2 University of Potsdam, Institute of Physics \& Astronomy, Karl-Liebknecht-Strasse 24/25, 14476 Potsdam, Germany \\ 3 University of Chicago, Department of Astronomy\&Astrophysics, 5640 S Ellis Ave, AAC 010c, Chicago, IL 60637, USA
}

Received 15 November 2012 / Accepted 12 February 2013

\begin{abstract}
Context. Supernova remnants (SNRs) are thought to be the primary candidates for the sources of Galactic cosmic rays. According to the diffusive shock acceleration theory, SNR shocks produce a power-law spectrum with an index of $s=2$, perhaps nonlinearly modified to harder spectra at high energy. Observations of SNRs often indicate particle spectra that are softer than that and show features not expected from classical theory. Known drawbacks of the standard approach are the assumption that SNRs evolve in a uniform environment, and that the reverse shock does not accelerate particles. Relaxing these assumptions increases the complexity of the problem, because one needs reliable hydrodynamical data for the plasma flow as well as good estimates for the magnetic field $(\mathrm{MF})$ at the reverse shock.

Aims. We show that these two factors are especially important when modeling young core-collapse SNRs that evolve in a complicated circumstellar medium shaped by the winds of progenitor stars.

Methods. We used high-resolution numerical simulations for the hydrodynamical evolution of the SNR. Instead of parametrizations of the MF profiles inside the SNR, we followed the advection of the frozen-in MF inside the SNR, and thus obtained the B-field value at all locations, in particular at the reverse shock. To model cosmic-ray acceleration we solved the cosmic-ray transport equation in test-particle approximation.

Results. We find that the complex plasma-flow profiles of core-collapse SNRs significantly modify the particle spectra. Additionally, the reverse shock strongly affects the emission spectra and the surface brightness.
\end{abstract}

Key words. ISM: supernova remnants - cosmic rays - magnetic fields - hydrodynamics - shock waves radiation mechanisms: non-thermal

\section{Introduction}

It is often assumed that cosmic rays (CRs) with energies below the knee of the CR spectrum $\left(\sim 10^{16} \mathrm{eV}\right)$ are accelerated in supernova remnants (SNRs). If this were correct, particle spectra should follow a power-law with index, $s=2$, with an exponential cut-off at very high energy. However, the wealth of recent observational data on, e.g., RX J0852.0-4622 (Aharonian et al. 2007), RCW 86 (Aharonian et al. 2009), SN 1006 (Acero et al. 2010), Cas A (Acciari et al. 2010; Abdo et al. 2010), and Tycho's SNR (Acciari et al. 2011; Giordano et al. 2012) provide strong evidence that the particle spectra are significantly softer. Moreover, the spectral shape is not a pure power law with exponential cut-off, as for example in Cas A. In addition, multiwavelength images of SNRs puzzle observers and force them to introduce multi-zone emission models (Araya \& Cui 2010; Lemoine-Goumard et al. 2012; Atoyan \& Dermer 2012).

Several groups have introduced modifications to classical diffusive shock acceleration (DSA) theory (Axford et al. 1977; Krymskii 1977; Bell 1978; Blandford \& Ostriker 1978) and its nonlinear counterpart (NDSA) (Malkov \& O'C Drury 2001). Malkov et al. (2012) claim that ion-neutral collisions in the SNR vicinity may steepen the energy spectrum of particles by one power with respect to classical DSA due to an evanescence of Alfvén waves that permits the escape of particles in a certain momentum range. Blasi et al. (2012) discuss the spectral softening that arises from the energy and momentum transfer of neutrals that return to the upstream region. The mean free path of neutrals is longer than the length-scale of shock modification because of $\mathrm{CR}$ streaming, and hence the effective compression ratio is $<4$ and the particle spectrum is soft even for high-energy particles. Caprioli (2012) argues that in the upstream region the scattering centers propagate with Alfvén velocity, which in the presence of very strong MF amplification reduces the compression ratio and leads to soft spectra of particles (see also Lerche et al. 2000). According to this study, the acceleration efficiency saturates at around $30 \%$. Inoue et al. (2010) consider multiple weak secondary shocks that appear when the primary forward shock propagates through a medium filled with small dense cloudlets. The CRs are re-accelerated at these low-Mach-number shocks, resulting in a softer spectrum. The largest and strongest secondary shock in the system is the reverse shock (RS) that propagates through the ejecta. High-resolution radio and X-ray observations support the notion that particle acceleration can also occur at RS (Gotthelf et al. 2001; Rho et al. 2002; DeLaney et al. 2002; Sasaki et al. 2006; Helder \& Vink 2008), but only recently has this possibility been considered in theoretical calculations (Zirakashvili \& Aharonian 2010; Zirakashvili \& Ptuskin 2012; Telezhinsky et al. 2012a,b), which relax the restricting assumptions about the magnetic field (MF) in the RS region (e.g. Ellison et al. 2005). The RS accelerates particles of the ejecta and thus provides a second population of relativistic particles 
in addition to that produced at the forward shock. The superposition of these two components may lead to spectral modifications in the volume-integrated emission from SNRs that are not easily reproduced with so-called one-zone/one-shock models, and accordingly, multiple zones/particle populations need to be employed (Araya \& Cui 2010; Lemoine-Goumard et al. 2012; Atoyan \& Dermer 2012). Misinterpretations are possible if the forward shock (FS) is considered the sole accelerator.

Another idealization is the assumption that SNRs evolve in a uniform environment. Given that stellar mass-loss considerably modifies the surroundings of stars, as well as known inhomogeneities in the interstellar medium, it is clear that this cannot hold, and calculations have started to take into account the complicated nature of the SNR environment (Ellison \& Bykov 2011; Zirakashvili \& Ptuskin 2012; Ellison et al. 2012; Telezhinsky et al. 2012b). This increases the complexity of the problem, because one requires reliable hydrodynamics of the plasma flow as well as knowledge about the MF at the reverse shock.

In this paper we show that taking into account both points, namely the acceleration of particles at the RS and the complex hydrodynamics of SNRs, is particularly important for modeling young core-collapse SNRs that evolve in a circumstellar medium shaped by the wind from the progenitor star. The resulting volume-integrated spectrum of particles and their consequent radiation is significantly different from that of planar-shock calculations and dependent on the type of SNR. To accomplish our study, we perform high-resolution simulations of the hydrodynamical evolution of the SNR with initial and environmental conditions representative of type Ic and type IIP supernovae ( $\mathrm{SNe}$ ). We consider the transport of frozen-in MF by the plasma flow inside the SNR to trace its evolution inside the remnant and particularly in the RS region separately for the radial and the tangential field. We model cosmic-ray acceleration by solving the cosmic-ray transport equation in test-particle approximation. Finally, we calculate the resulting emission from the SNR and construct surface-brightness maps in various energy bands. Thus we trace the complex particle distribution resulting from particle acceleration at both shocks.

\section{Hydrodynamics}

Our goal is to study how the evolution of SNR shock waves into the medium created by their progenitor stars modifies the spectra and high-energy emission from these objects. Given the impossibility of exploring the total diversity of paths that can lead to a supernova explosion, we have instead chosen to sample two distinctive types of SNe. These illustrate the detailed structure of the circumstellar medium into which $\mathrm{SNe}$ advance, and also how the various features in the spectra, and the very high energy emission, relate to the evolutionary properties of the SN.

We investigate here type Ic and type IIP SNe. We chose these because they show two different regimes of evolution, and because many $\mathrm{SNe}$, even those of other types, will fall somewhere close to or in between these types. For instance, Cas A is classified as a type IIb SN based on spectra obtained from light echoes. However, its evolution in a red supergiant (RSG) wind (Chevalier \& Oishi 2003), within which it is probably currently expanding, would be quite similar to the evolution in a wind medium for a type IIP SN. Thus we hope to give an overview of the spectra and evolution of many different types of young SNRs via these calculations. Below we describe the assumptions that went into the simulations, and the evolution of the remnant in each case.

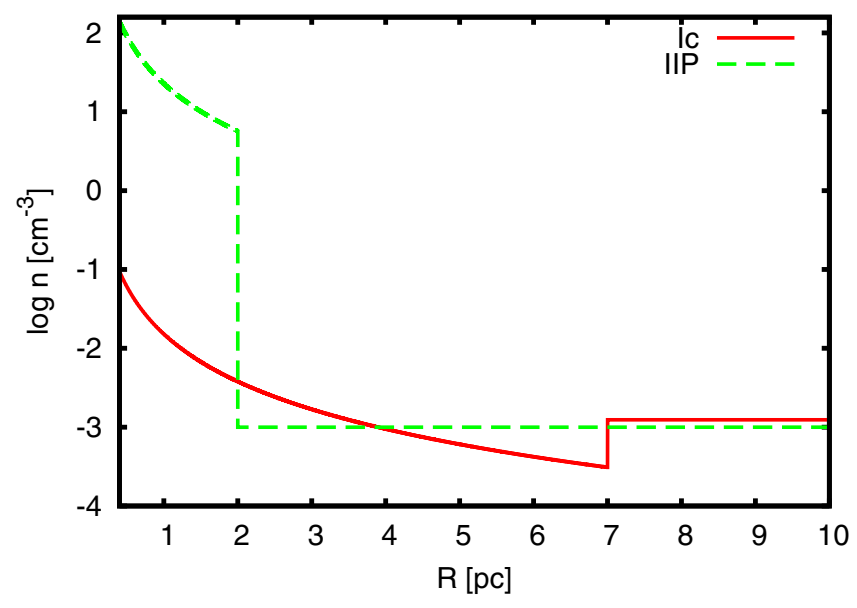

Fig. 1. Density profiles of circumstellar gas before the explosion of type-Ic and type-IIP SNe.

To simulate the SN expansion into the ambient medium, we first need to understand the nature of the ambient medium. Since the medium around core-collapse $\mathrm{SNe}$ is shaped mainly by mass-loss from the progenitor star, we need to take into account the evolution of the progenitors of these SNe.

Type IIP SNe arise from the explosion of RSGs. In general, these stars start as O- or B-type stars while on the main sequence, with initial masses between about 8 and 30 solar masses. In the main-sequence stage these stars lose mass via radiatively driven winds, with high wind velocities $\left(>1000 \mathrm{~km} \mathrm{~s}^{-1}\right)$ with a mass-loss rate of about $10^{-7} M_{\odot} \mathrm{yr}^{-1}$. The interaction of the main-sequence wind with the surrounding (constant-density) interstellar medium gives rise to a wind-blown bubble (Weaver et al. 1977; Garcia-Segura et al. 1996; van Marle et al. 2005; Dwarkadas 2005, 2007; Toalá \& Arthur 2011; Dwarkadas \& Rosenberg 2013). The standard structure of the wind bubble, going outwards in radius from the star, consists of a freely expanding wind that ends in a wind termination shock, a hot, lowdensity shocked wind medium, a contact discontinuity, outer shock, and the external medium. If the wind has constant parameters, the freely expanding wind will have a density profile that decreases as $r^{-2}$, while the shocked wind will have a more or less constant density. It is possible that, depending on the surface temperature and number of ionizing photons emitted from the star, there is a dense ionized (HII) region inside the constant discontinuity (see e.g. Dwarkadas 2011). Since this does not happen in all cases, we have not taken it into account.

As the star moves off the main sequence into the RSG stage, it grows considerably in size, the wind mass-loss rate increases to about $5 \times 10^{-5} M_{\odot} \mathrm{yr}^{-1}$ while the velocity drops to a low value of about $10 \mathrm{~km} \mathrm{~s}^{-1}$. This results in a new pressure equilibrium. The high density $\left(\propto \dot{M} / v_{w}\right)$ of the RSG wind leads to the formation of a wind region with density almost four orders of magnitude above that of the main-sequence wind. The RSG wind can also end in a shell, but we chose for simplicity to exclude this. This leads to the density structure shown in Fig. 1, with an initial high-density wind with density decreasing as $r^{-2}$, followed by a steep drop in density toward the main-sequence wind zone, ending in the main-sequence shell.

If the initial mass of the star exceeds about $30 M_{\odot}$ (depending on which models are used), the star will not end its life as a RSG. It will leave the main sequence to become an RSG, or, in stars with initial mass $>50 M_{\odot}$, to become a luminous blue variable. Following this, it may shed the outer $\mathrm{H}$ and perhaps 

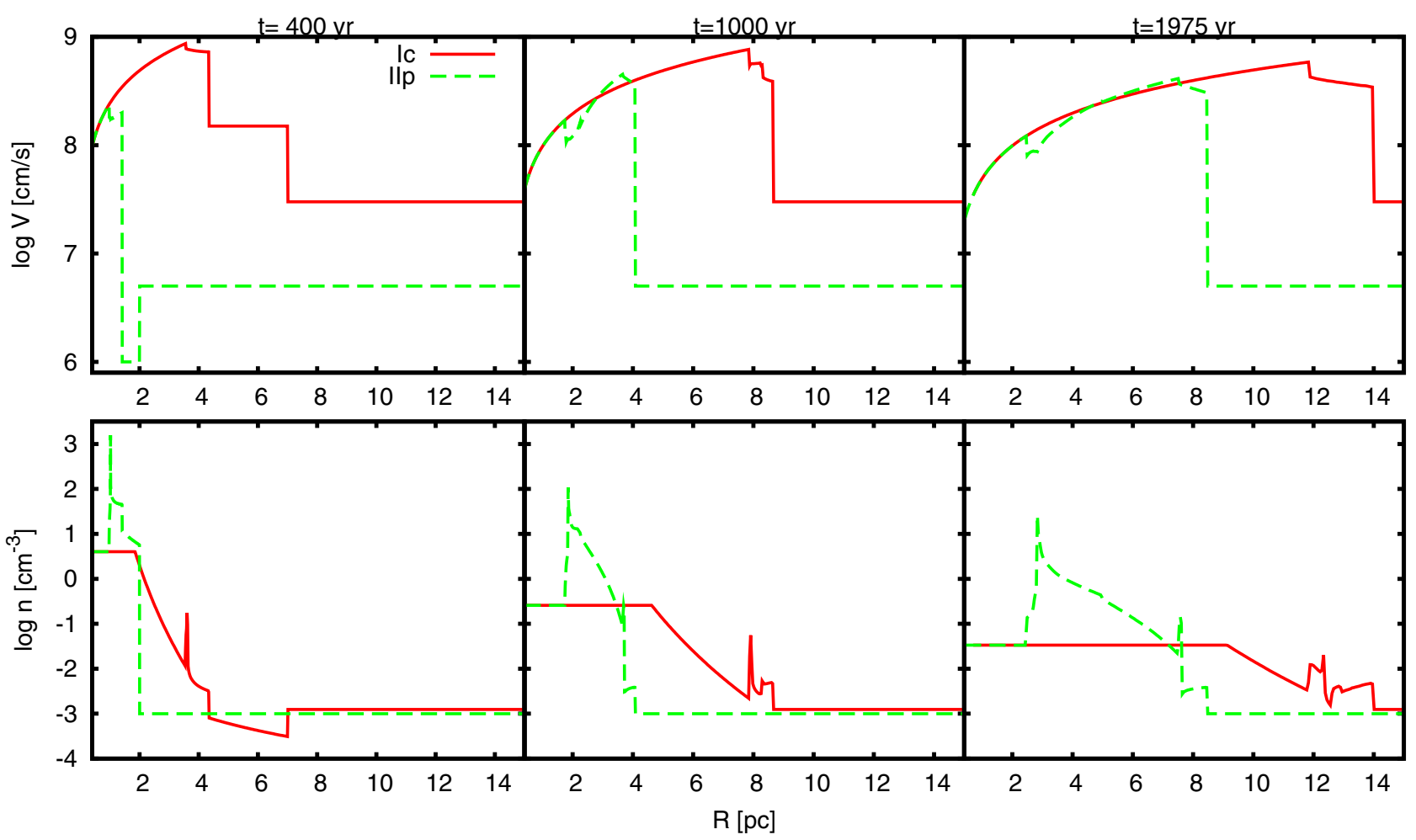

Fig. 2. Time evolution of the plasma outflow velocity (top) and density (bottom) as a function of radius for type-Ic and type-IIP SNRs.

He envelope, turning into a Wolf-Rayet (WR) star. The mass-loss rates of WR stars are somewhat lower than those of RSG winds, but their wind velocities are more than two orders of magnitude higher, leading to wind densities that are more than two orders of magnitude lower. The high momentum of the winds pushes outward on the RSG shell, breaking it up in the process and mixing the RSG material into the WR wind (Dwarkadas 2007; Toala \& Arthur 2011; Dwarkadas \& Rosenberg 2013). This mixed material approaches, and could bounce back from, the main-sequence shell. Eventually, the system reaches an equilibrium situation that in many ways resembles the main-sequence bubble (and has almost the same radius, since the main sequence-shell is very dense and expanding very slowly, on the order of $20-50 \mathrm{~km} \mathrm{~s}^{-1}$ ), but the extent of the freely expanding wind and shocked wind, and the radius of the wind termination shock, are different. This leads to the situation shown in Fig. 1.

To explore the situation without having to simulate the entire evolution of the progenitor star and its wind medium, we approximated the wind density profile using reasonable average parameters. Following this, we investigated the evolution of the SN shock wave within the medium. We used the VH-1 code, a 3D finite-difference hydrodynamic code based on the piecewise parabolic method (Colella \& Woodward 1984). Since we aim to study the effects of the complicated environment, we assumed similar characteristics for the SN density structure in each case, so that the differences in the evolution result purely from the difference in the ambient medium. In each case we assumed an ejecta mass of about $5 M_{\odot}$ and an explosion energy of $10^{51} \mathrm{erg}$. The ejecta density is flat where the plasma flow velocity is below a certain value, $u_{\mathrm{fl}}$, and decreases as a power law with radius, $\rho_{\mathrm{ej}} \propto r^{-9}$, where the flow velocity is above $u_{\mathrm{fl}}$ (Chevalier \& Fransson 1994; Dwarkadas 2005). The interaction of the ejecta with the wind medium sets up a double-shock structure as expected, consisting of a forward and reverse shock separated by a contact discontinuity. The region between the outer shock and contact discontinuity contains shocked surrounding medium, whereas the region between the inner shock and contact discontinuity includes shocked ejecta. The structure of this shocked region depends on the ejecta density and the density profile of the surrounding medium (Chevalier 1982; Dwarkadas 2011), and has implications for the emission from the remnant.

The shock expansion in the wind medium in both cases is quite similar initially, except that the shock moves slower in the high-density region, as expected. The evolution changes once the shock reaches the end of the freely expanding wind region. In the RSG case one finds a huge drop in density beyond the wind region, whereas in the WR case one finds an increase in density by a factor of 4 . This change in density and the transition from the wind zone to a medium of almost constant density destroys the self-similarity of the solution. The interaction of the SNR forward shock with the wind termination shock leads to a reflected shock that travels back into the ejecta in the case of the type Ic SN. In the case of the type IIP, the steep drop leads to the formation of a complicated ejecta structure. These structures are used to compute the acceleration of particles at the shock fronts. The evolution of the plasma velocity and density profiles is shown in Fig. 2.

\section{Magnetic field}

The acceleration and subsequent radiation of relativistic particles significantly depends on the distribution of MF in the SNR, especially in the upstream and downstream vicinities of the shocks. In our previous work (Telezhinsky et al. 2012a,b), we assumed parametrizations for the MF inside the remnant following either pressure or density distributions. We found that these simple parametrizations fail for type-IIP SNRs, since the energy contained in MF would be roughly equivalent to the explosion energy after a few hundreds years of evolution. In this work we implement a more realistic description of the MF distribution 
inside SNRs, which is especially important for the acceleration of particles at the RS, a key ingredient of our studies.

We start with the assumption that the SNR is filled with a perfectly conducting fluid, which is common in astrophysical plasmas. The evolution of the frozen-in MF satisfies the equation

$$
\frac{\partial \boldsymbol{B}}{\partial t}=\nabla \times(\boldsymbol{u} \times \boldsymbol{B})
$$

where $\boldsymbol{B}$ is the MF and $\boldsymbol{u}$ is the flow speed. Rewriting the cross product in Eq. (1) in terms of dot products gives

$$
\frac{\partial \boldsymbol{B}}{\partial t}=\boldsymbol{u}(\nabla \cdot \boldsymbol{B})-\boldsymbol{B}(\nabla \cdot \boldsymbol{u})+(\boldsymbol{B} \cdot \nabla) \boldsymbol{u}-(\boldsymbol{u} \cdot \nabla) \boldsymbol{B} .
$$

Since we have spherically symmetric flow, $u_{r}=u_{r}, u_{\theta}=0$, $u_{\phi}=0$ and $\partial u_{r} / \partial \theta=0, \partial u_{r} / \partial \phi=0$. Setting $\theta=\pi / 2$, i.e., $B_{\theta}$ and $B_{\phi}$ scale in the same fashion and together form the tangential field, and using

$\nabla \cdot \boldsymbol{B}=0=\frac{1}{r^{2}} \frac{\partial\left(r^{2} B_{r}\right)}{r}+\frac{1}{r} \frac{\partial B_{\theta}}{\partial \theta}+\frac{1}{r} \frac{\partial B_{\phi}}{\partial \phi}$,

we reduce Eq. (2) (see appendix) to a set of expressions that describe the evolution of the radial and tangential components of $\boldsymbol{B}$,

$$
\begin{aligned}
& \frac{\partial B_{r}}{\partial t}=-\frac{\partial}{\partial r}\left(B_{r} u_{r}\right)-2 B_{r} \frac{u_{r}}{r}+B_{r} \frac{\partial u_{r}}{\partial r} \\
& \frac{\partial B_{\theta}}{\partial t}=-\frac{\partial}{\partial r}\left(B_{\theta} u_{r}\right)-B_{\theta} \frac{u_{r}}{r} \\
& \frac{\partial B_{\phi}}{\partial t}=-\frac{\partial}{\partial r}\left(B_{\phi} u_{r}\right)-B_{\phi} \frac{u_{r}}{r}
\end{aligned}
$$

Eq. (4) represents the transport of the MF components with the flow. To solve them, we need to apply initial values and boundary conditions.

In the core-collapse type-IIb SN 1993J, the MF in the emission region is found to be $\simeq 64 \mathrm{G}$ at an age of a few days, when the SNR has a radius of roughly $R_{\mathrm{SN}}=10^{15} \mathrm{~cm}$ (Fransson \& Björnsson 1998; Martí-Vidal et al. 2011). Given the resolution of the radio data, it is not clear whether the emission region in such a young $\mathrm{SN}$ is decoupled from the ejecta or not. Therefore we interpret this MF as the average field of the SN and valid also for the ejecta. There are several arguments supporting this interpretation. (i) During the collapse of a massive star, a number of processes such as magneto-rotational (Akiyama et al. 2003) and stationary-accretion-shock (Endeve et al. 2010) instabilities, small-scale dynamo action (Thompson \& Duncan 1993), Alfvén-wave amplification (Guilet et al. 2011), or simple winding of MF lines in differential rotation may result in a significant amplification of the MF (Obergaulinger \& Janka 2011). The amplified field may be ejected outward together with part of the stellar material in the supernova explosion (Endeve et al. 2010, 2012; Obergaulinger \& Janka 2011). The expansiondiluted MF will be roughly as strong as observed in SN 1993J. (ii) Common sense suggests that the outflow of ejecta is not laminar. Therefore, various turbulence-induced MF-amplification processes (Beresnyak et al. 2009; Guo et al. 2012; Sano et al. 2012) may operate also at the RS propagating through the ejecta. Alternatively, it may be that the high observed MF is amplified by CR-streaming instabilities. In the latter case, however, inserting parameter values found by Fransson \& Björnsson (1998); Martí-Vidal et al. (2011) into formulas for the saturation level (Caprioli et al. 2009; Luo \& Melrose 2009) yields values of the MF strength that are between one (non-resonant mode) and two (resonant mode) orders of magnitude lower than observed. Additionally, the time evolution of MF will not be as observed (Dwarkadas et al. 2012).

To ensure that initially $\nabla \cdot \boldsymbol{B}=0$ in the ejecta, we assume that $B_{\mathrm{ej}, \mathrm{r}}$ is flat where $u_{r} \leq u_{\mathrm{fl}}$ and $B_{\mathrm{ej}, \mathrm{r}}(r) \propto 1 / r^{2}$ where $u_{r}>u_{\mathrm{fl}}$. We assume the same for the tangential field, and scale all components so that the total volume-averaged MF strength is $\simeq 50 \mathrm{G}$ when $R_{\mathrm{SN}}=10^{15} \mathrm{~cm}$. For the initial condition at $t_{0}=1$ year, we use the MF scaled according to flux conservation:

$$
B_{i}\left(r, t_{0}\right)=B_{\mathrm{ej}}(r) \frac{R_{\mathrm{SN}}^{2}}{R_{\mathrm{SNR}}\left(t_{0}\right)^{2}} .
$$

In the current work we limit ourselves to scenarios with no amplification of the MF upstream of the FS, and only the CSM field is assumed to be transported through the shock to the downstream region. The MF in the circumstellar medium (CSM) of type-Ic and type-IIP SNRs is shaped by the winds of the progenitor stars. We assume that turbulence equalizes all components of the MF in the wind zones until they are transported through the FS. The profile of the MF in the environment of a core-collapse SNR in our description is then given by

$B(r)= \begin{cases}B_{\mathrm{b}}(r) & R_{\mathrm{st}} \leq r \leq R_{\mathrm{b}} \\ B_{\mathrm{b}}\left(R_{\mathrm{b}}\right) \sqrt{11} & R_{\mathrm{b}} \leq r \leq R_{\mathrm{sh}},\end{cases}$

where $B_{\mathrm{b}}(r)=B_{\mathrm{st}} R_{\mathrm{st}} / r, B_{\mathrm{st}}$ is the MF at the surface of the progenitor star $\left(\approx 100 \mathrm{G}\right.$ for WR stars, $\approx 1 \mathrm{G}$ for RSGs), $R_{\mathrm{st}}$ is the radius of the progenitor star $\left(\approx 8 R_{\odot}\right.$ for WR star, $\approx 600 R_{\odot}$ for $\mathrm{RSG}), R_{\mathrm{b}}$ is the radius of the wind-blown bubble in our simulations ( $\approx 7 \mathrm{pc}$ for WR star, $\approx 2 \mathrm{pc}$ for $\mathrm{RSG}$ ), $R_{\mathrm{sh}}$ is the radius of the swept-up shell $(\approx 30 \mathrm{pc}$ in both cases $)$, and $r$ is the distance from the star. Note that the $1 / r$ scaling of the field strength results from the advection of the stellar MF in a wind, colloquially known as the Parker spiral, where it applies to the tangential components only. The radial field in the wind zone is supposed to arise from turbulence, which may in fact also amplify it (Drury \& Downes 2012), and should be understood as RMS amplitude. Magnetic-field amplification by cosmic-ray streaming (Lucek \& Bell 2000), upstream dynamo action (Beresnyak et al. 2009), the Richtmeyer-Meshkov instability (Sano et al. 2012), or vorticitygeneration at shocks (Giacalone \& Jokipii 2007) are not considered here.

The solution of Eq. (4) is obtained numerically, using the initial conditions described above and the parameters of the SNR plasma flow from the simulations described in Sect. 2. The timedependent solution for different SNR types is plotted in Fig. 3. Our solutions for the MF evolution are consistent with earlier 1D studies (Rosenau \& Frankenthal 1976), but 2D MHD solutions (Schure et al. 2009) show significant distortions at the contact discontinuity due to the growth of the Rayleigh-Taylor (or Kruskal-Schwarzschild) instability.

\section{Particle acceleration method}

Our method is based on time-dependent kinetic calculations in a test-particle approximation (Telezhinsky et al. 2012a,b). They are implemented by numerical solution of the diffusionadvection equation for the differential particle number density on a grid co-moving with the shock wave and in spherically symmetric geometry. To resolve the diffusion length of the lowest energy particles, we performed a coordinate transformation that increases the spatial resolution near the shock. The spatial 


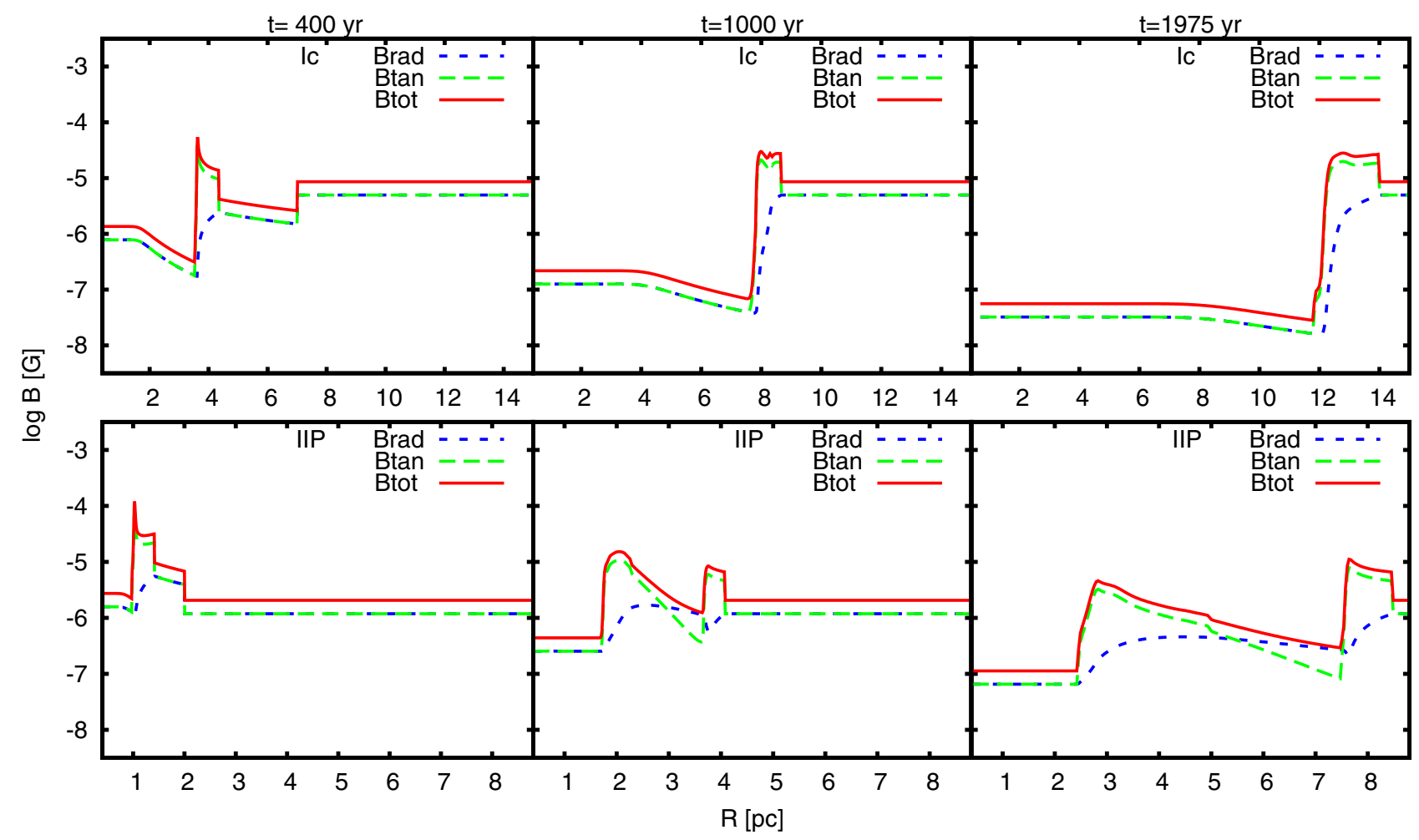

Fig. 3. Time evolution of the total, tangential, and radial MF as a function of radius for type-Ic (top) and type-IIP (bottom) SNRs.

coordinate, $x$, is related to the new coordinate, $x_{*}$, for which a uniform grid is used, by the equation

$(x-1)=\left(\frac{r}{R_{\mathrm{SH}}}-1\right)=\left(x_{*}-1\right)^{3}$,

where $R_{\mathrm{SH}}$ is the shock radius. Thus, with modest resolution in the $x_{*}$, one may achieve a very fine resolution in $x$ where it matters, namely in the shock region where the newly injected lowmomentum particles enter the acceleration process. The other benefit is the significant extension of the grid toward $x \gg 1$ at very low computation cost, since the range in $x$ is proportional to the third power of $x_{*}$. The boundary condition for large $x$ then does not really affect our solution, since particles do not leak out of the grid but rather are distributed over the huge upstream volume according to the diffusion properties of the media. We included an exponential transition from Bohm to Galactic diffusion at around $2 R_{\mathrm{FS}}$. A high Galactic diffusion coefficient also compensates for the deteriorating resolution of the spatial grid beyond $2 R_{\mathrm{FS}}$. Because our method is based on the test-particle approximation, we did not permit the CR pressure at the shock to exceed $10 \%$ of the ram pressure. We used a thermal-leakage injection model (Blasi et al. 2005), and adjusted the injection so that the CR-pressure limit was not violated. The injection coefficient, a free parameter, was taken to be approximately $5 \times 10^{-6}$ for type-Ic and for $5 \times 10^{-8}$ type-IIP SNR.

\section{Results and discussion}

Based on the hydrodynamic simulations described in Sect. 2 and the MF profiles obtained in Sect. 3, we compute timedependent particle distributions accelerated by the forward and reverse shocks. We calculate the resulting emission due to synchrotron, inverse Compton, and pion-decay processes, build corresponding surface-brightness maps and discuss the observational implications. We present snapshots at the age of 400, 1000, and 1975 years to trace spectral and morphological evolution of emission coming from type-Ic and type-IIP SNRs.

\subsection{Particle spectra}

Particle spectra for type-Ic and type-IIP SNRs are given in Fig. 4. We show spectra produced by acceleration at the forward and reverse shocks plotted along with a volume-integrated total spectrum from the whole SNR.

At an age of 400 years, the type-Ic SNR is still propagating through the freely expanding stellar wind. The flow velocity and density profiles are given by the classical self-similar solutions. The spectrum of protons accelerated by the FS is well described by a power-law with exponential cutoff, in agreement with classical DSA solutions. The magnetic field at the FS is still strong at this age, and so particles reach high energies. The spectrum of RS-accelerated protons is also consistent with standard DSA. Note that the volume integration was performed over both the upstream and the downstream region of the RS, whereas the upstream region of the FS was not covered (for details see Sect. 5.1.1 of our previous paper Telezhinsky et al. 2012a). The magnetic field at the RS is considerably smaller than at the FS, and so particle energies reach a TeV only. However, the number of particles injected into the acceleration process, and hence the density, is larger. Therefore, below a few TeV the CR number density at the RS is higher than that at the FS, and the total volume-integrated proton spectrum displays a downward step around a few TeV. In phenomenological studies of SNRs such spectra may be interpreted as the sum of different populations of particles coming from different zones of the remnant (Araya \& Cui 2010; Atoyan \& Dermer 2012; Lemoine-Goumard et al. 2012). Since the MF is not strong enough at either of the shocks 


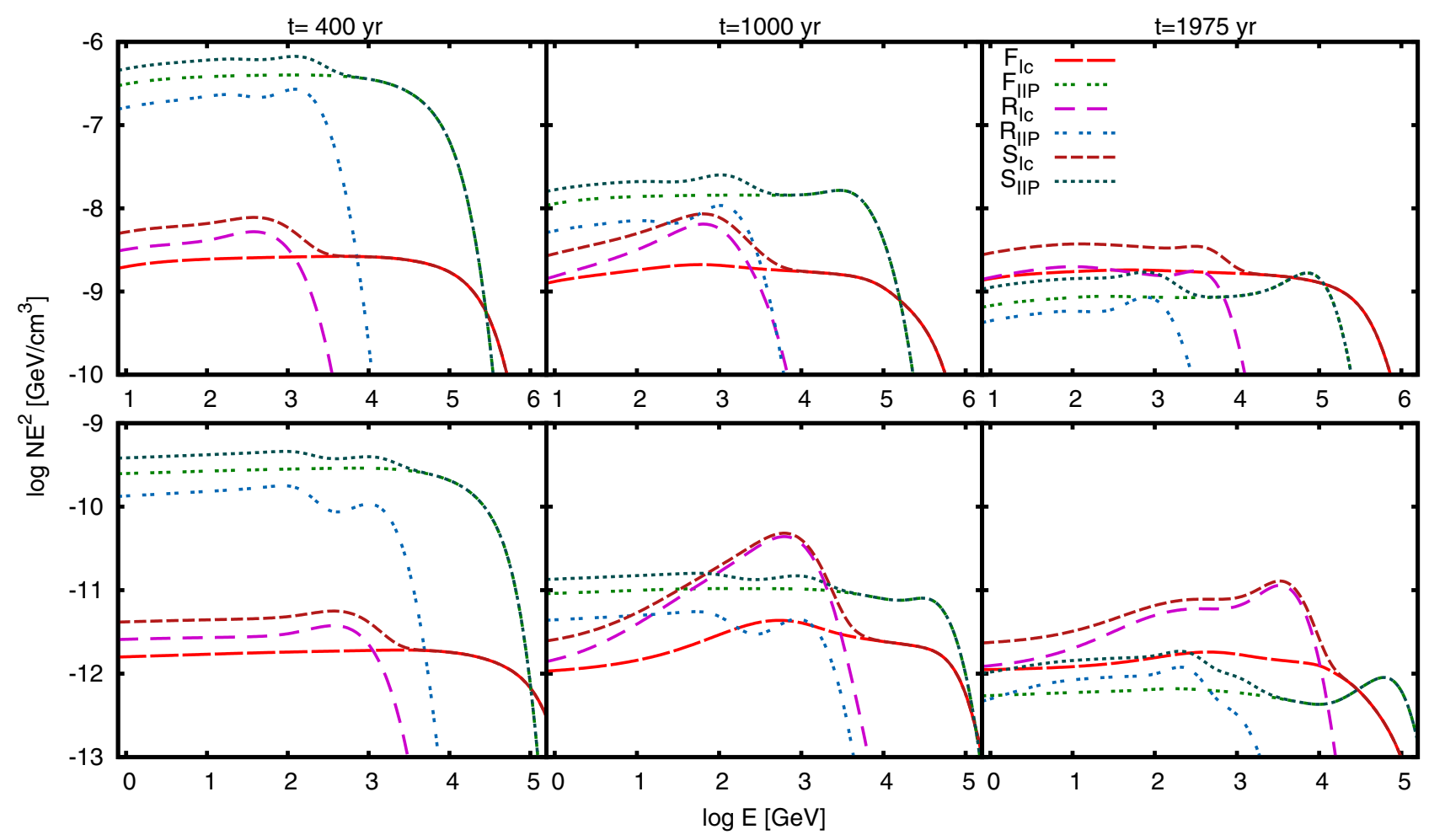

Fig. 4. Time evolution of the proton (top) and electron (bottom) spectra for type-Ic and type-IIP SNR. Total volume-integrated spectra (S) are the sum of the contributions from the forward $(\mathrm{F})$ and reverse $(\mathrm{R})$ shocks.

to cause significant synchrotron losses, the electron spectra look similar to those of protons.

The type-IIP SNR at the same age also evolves in the freely expanding progenitor wind, though this wind has a much higher density. Therefore both electron and proton spectra have a much higher intensity than in the type-Ic SNR. The density at the RS of the type-IIP SNR is lower than at the FS, and consequently the $\mathrm{CR}$ intensity is lower. Nonetheless, the contribution of the RS to the overall particle spectrum is noticeable. The magnetic field at the RS of the type-IIP SNR is slightly larger than in the type-Ic case because the radius is smaller, and particles are accelerated to somewhat higher energies, cutting off at around a few TeV. For the same reason, electron losses in the downstream region of the $\mathrm{RS}$ are more efficient and steepen the electron spectra beyond a characteristic energy, $E_{\mathrm{rad}}$, downstream of the RS, whereas in the upstream region of the RS the MF, and hence the electron energy losses, are weak. Since the volume-integrated spectrum of particles produced by the RS includes both the downstream and the upstream contributions, we observe a bump located at $E_{\max }$ of the RS upstream electron distribution, which is located beyond $E_{\text {rad }}$ (see Sect. 5.1.1 of Telezhinsky et al. 2012a for details).

At 1000 years, type-Ic SNR particle spectra produced by the FS differ significantly from those expected via standard DSA. The significant spectral modification can be traced back to the interaction of the FS with the wind termination shock, at around 700 years. The effective compression ratio at the FS as seen by the CRs increased significantly. The acceleration was boosted for a short time, and since the interaction time was brief and the spectral index hard, a bump at a few hundred GeV formed, which slowly moved toward higher energies as the particles in it were further accelerated by the FS.

By the time of 1000 years, a break at around $1 \mathrm{TeV}$ is still clearly visible. When the FS collided with the wind termination shock, a reflected shock that propagates inward formed.
By coincidence, the time when the reflected shock reaches the $\mathrm{RS}$ of the remnant is very close to 1000 years. So the RS spectra shown are rather specific for this particular time, but we show them to illustrate the impact of the hydrodynamics on particle spectra. In analogy with the FS, the compression ratio at the RS increased for a very brief time of interaction, the spectral index became very hard, and a bump at a few tens of $\mathrm{GeV}$ formed and shifted to a few hundred $\mathrm{GeV}$ by the time of 1000 years.

Compared to the type-Ic, type-IIP SNRs do not show such a strong variation in particle spectra at the same age. The forward shock of the type-IIP SNR encountered the MS wind zone at around 600 years. Since the shock entered a very dilute environment, no significant additional compression occurred at the FS, but rather a very brief de-compression that did not affect $\mathrm{CR}$ spectra. After the encounter, another reverse shock formed and propagated through the FS-compressed CSM, and so we see a three-shock structure: the FS propagating through the MS wind zone, the internal RS going through the ejecta, and another reverse shock between them propagating through the dense CSM compressed earlier by the FS. Although the divergence of the plasma flow velocity is negative between the forward and intermediate shock, thus permitting re-acceleration of particles in this region and at the intermediate shock itself, we cannot see the effect in volume-integrated spectra. The FS speed increased when it entered the diluted-wind zone. The maximum energy of CRs, $E_{\max }$, increased as expected, but the rate of particle injection dropped significantly. The separation between the forward and reverse shocks started to increase on account of the boosted velocity of the FS, and so the volume enclosed by the RS constitutes a diminishing fraction of the total SNR volume. Nevertheless, the contribution of the RS-accelerated particles remains visible because the RS propagates through very dense ejecta. 


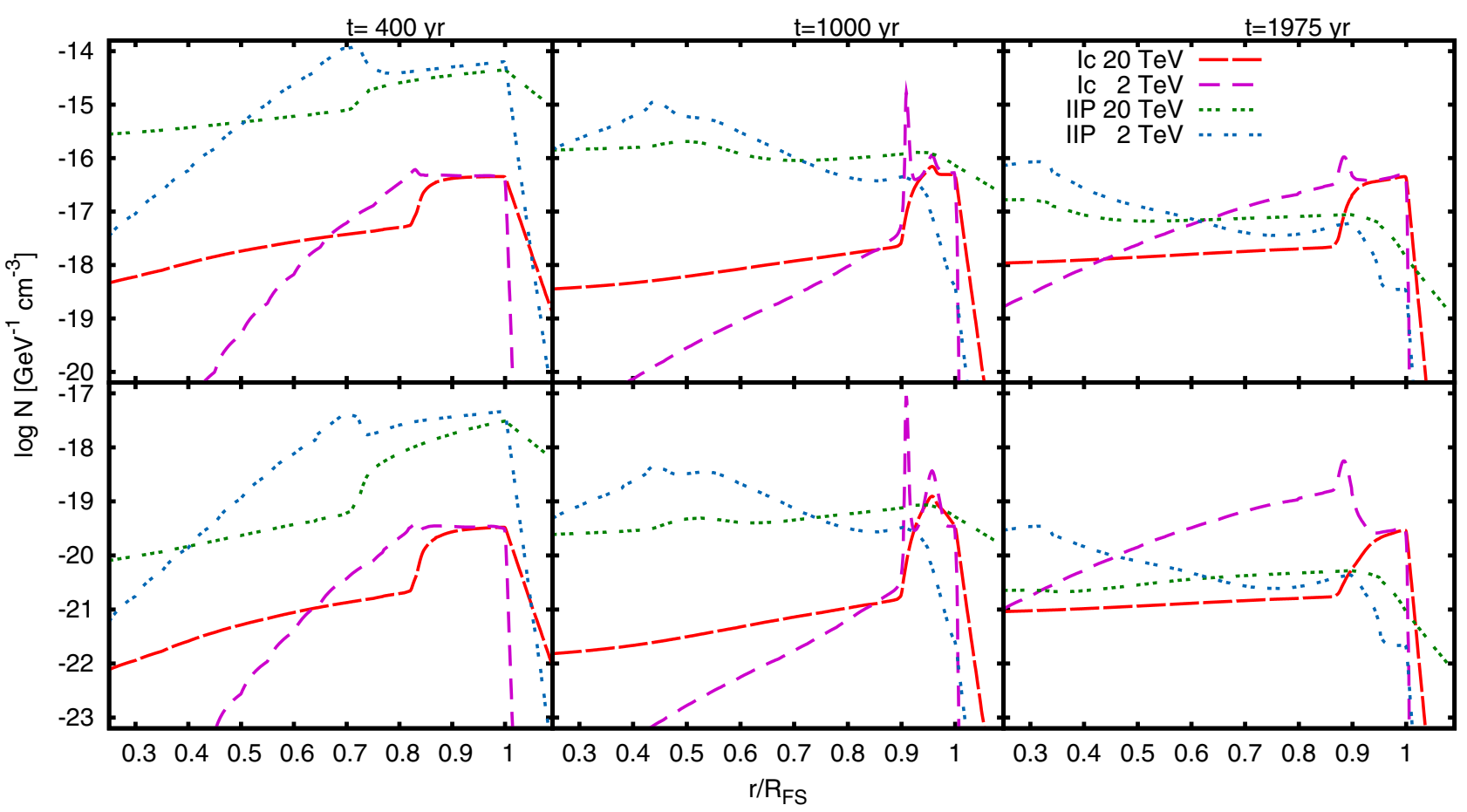

Fig. 5. Time evolution of the radial distribution of protons (top) and electrons (bottom) at an energy of $2 \mathrm{TeV}$ and $20 \mathrm{TeV}$ for different SNR types and models. The $2-\mathrm{TeV}$ distributions were downscaled by a factor 100 for better visualization.

Nearly 1000 years later, at the age of 1975 years, the velocity profiles of both remnant types have relaxed. The multiple weak reflected shocks in type-Ic SNR have dissipated, but the CR spectra still retain their effects, though in general they appear smoother than before. The proton distributions from both the FS and the RS are softer, and the contribution from the RS to the total proton spectra remains visible. The electrons accelerated by the FS have rather soft spectra as well, while those accelerated by the RS dominate the total electron spectra of type-Ic SNR.

At an age of 1975 years, we see an excess of high-energy particles accelerated by the FS in the type-IIP SNR. The electron spectra differ only slightly from the proton spectra due to weak synchrotron losses. Once the FS entered the dilute zone, the particle injection rate dropped four orders of magnitude. It takes time for the particle spectra to adjust to the new equilibrium, particularly so at very high energies. For the high-energy particles the characteristic diffusion-advection timescale, $\tau_{\mathrm{da}}=\kappa / v_{\mathrm{s}}^{2}$, is long, and therefore they continue to diffuse around the shock and gain energy while the lower-energy particles were advected from the shock. We can consider the bump as a signature of the delayed reaction of the high-energy particles to a sudden change in the injection rate.

\subsection{Radial distributions}

The radial distributions of $2-\mathrm{TeV}$ and $20-\mathrm{TeV}$ particles are presented at Fig. 5. There is a clear difference between particle profiles depending on both energy and SNR type.

The 20-TeV particle profiles comprise only particles accelerated at the FS, because the RS does not accelerate CRs up to this energy on account of the small MF. On the other hand, the RS region is prominent in the $2-\mathrm{TeV}$ particle profiles because at this energy CRs do not efficiently propagate away from their acceleration sites. Since cosmic-ray electrons lose energy much faster than protons, their intensity falls very quickly with increasing distance from the shocks.
At the age of 400 years, type-Ic SNRs show an increased number density of $20-\mathrm{TeV}$ particles between the two shocks. There, the MF is higher than in other parts of the SNR, and so particles are retained. In the ejecta region, where the MF is low, particles are distributed rather uniformly with an intensity approximately an order lower than in the shocked region. The intensity of $2-\mathrm{TeV}$ particles shows an enhancement in the region of the RS on account of the RS acceleration, but only marginally so because the cutoff energy of particles accelerated at the RS is below $2 \mathrm{TeV}$. A steep decrease of intensity toward the SNR center arises because the diffusion time of $2-\mathrm{TeV}$ particles is long and the age of the SNR is insufficient to distribute 2-TeV particles uniformly.

The profiles of 20-TeV particles in type-IIP SNRs of the same age behave similarly. The intensity of $2-\mathrm{TeV}$ CRs shows a significant peak at the RS, though, because the MF at the reverse shock of type-IIP SNR is stronger than in type-Ic SNR. The maximum energy of RS-accelerated particles is therefore considerably higher than in the case of type-Ic SNRs, in fact it is well beyond $2 \mathrm{TeV}$, thus leading to a prominent contribution of CRs from the RS.

As we mentioned above, the age of 1000 years happens to be a special phase in the evolution of type-Ic SNR for our choice of parameters, and it is interesting to see what happens to particle distribution just after the shocks collide. The profiles of $20-\mathrm{TeV}$ particle are largely unaffected by the recent interaction. The MF at the RS is insufficient to re-accelerate particles up to $20 \mathrm{TeV}$ and raise the intensity at this energy. A small peak in between two shocks can be explained by the increased MF at the contact discontinuity behind one of the weak reflected shocks; a similar structure is visible in the profiles of $2-\mathrm{TeV}$ particles. This MF creates a barrier for particle diffusion to the SNR center. The 2-TeV CR profiles are strongly affected by the recent shock collision. An excess of 2-TeV particles is clearly seen at the RS and, in fact, also in the volume-integrated particle spectra. Since the 


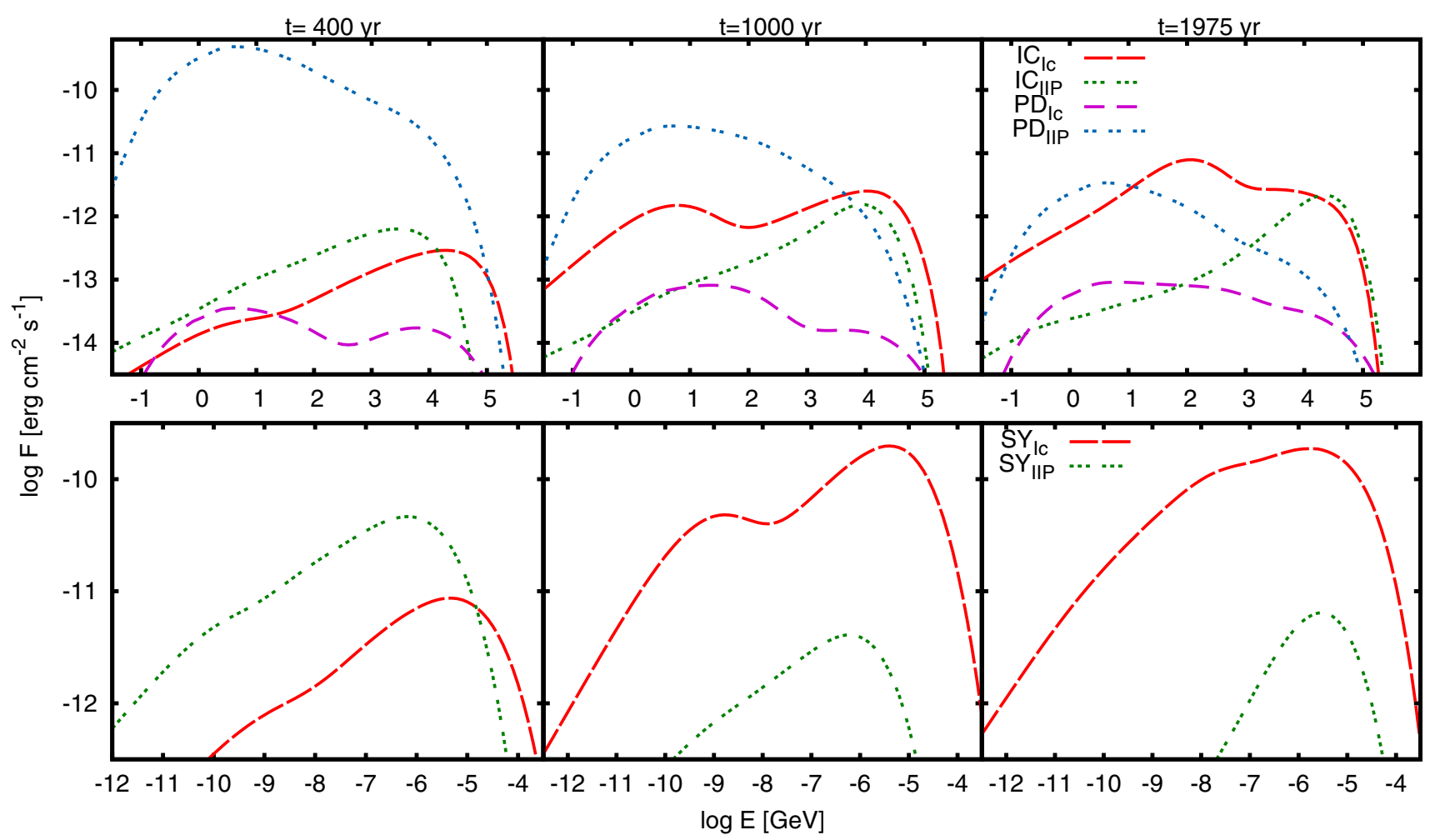

Fig. 6. Time evolution of the emission spectra from type-Ic and type-IIP SNRs due to pion-decay (PD), inverse Compton (IC), and synchrotron (SY) radiation.

particles have no time to diffuse around the acceleration region, the peak is very sharp.

After 1000 years, the radial distribution of CRs in type-IIP SNR has changed dramatically compared to a few hundred years earlier when the FS entered the MS wind zone. At the age of 1000 years the bulk of the CRs is relatively old, having been accelerated when the FS was propagating through the dense RSG wind. The high intensity of CRs, which were advected inside the SNR, joins smoothly with the low intensity of particles freshly accelerated by the FS. The profiles of $20-\mathrm{TeV}$ particles are significantly smoothed on account of fast diffusion, whereas the intensity of 2-TeV particles shows an increase toward the RS. The RS propagating through dense ejecta at $0.4 R_{\mathrm{FS}}$ strongly contributes to the profile of $2-\mathrm{TeV}$ particles.

After 1975 years, the particle profiles in type-Ic SNR have lost memory of the reflected shocks present earlier. The spatial distribution is rather smooth with enhanced intensity in the shocked region, at $2 \mathrm{TeV}$ particularly so at the RS suggesting that acceleration is still ongoing. Low-energy particles are far more abundant in the SNR interior than they were at early times. At $20 \mathrm{TeV}$ the intensity is nearly constant in the SNR interior.

The radial profiles of particles in type-IIP SNR have not changed much from 1000 to 1975 years. The profiles became smoother, but the peak at the RS at around $0.3 R_{\mathrm{FS}}$ is still observed for 2-TeV particles. Interestingly, the intermediate shock (see previous subsection) is now visible as an increase in lowenergy particle intensity at around $0.9 R_{\mathrm{FS}}$. Although no impact of the intermediate shock is seen in volume-integrated spectra, it clearly affects radial profiles, which suggests that some re-acceleration occurs between the forward and intermediate shocks in addition to particle trapping at the contact discontinuity. This effect is not as relevant for $20-\mathrm{TeV}$ particles on account of fast diffusion, and so their distribution is rather smooth.

\subsection{Nonthermal emission}

We considered three radiation processes of non-thermal particles: synchrotron and inverse Compton (IC) emission of electrons, and neutral pion decays originating in collisions of $\mathrm{CR}$ protons with protons at rest. Only primary electrons are considered for the leptonic processes. The calculated photon distributions for type-Ic SNR and type-IIP SNR are plotted at Fig. 6. We also calculate, and discuss in context with the radiation spectra, intensity maps for the emission at characteristic radio, X-ray and gamma-ray wavelengths. The maps are shown in Fig. 7. We note that in all calculations of volume-integrated spectra or intensity maps the radial distributions of CRs, the target material, and MF are taken into account. The result is significantly different from simple toy-model calculations assuming uniform particle, density, and MF profiles inside the SNR. We first give brief details on the method of calculation and continue with a discussion of our results.

The relevant MF component for synchrotron emission is that in the plane-of-sky, $B_{\perp}$, which is perpendicular to the line of sight (LOS). If all components of the MF are equal, then it is straightforward to show that the effective value of $B_{\perp} \simeq 0.8 B$. When properly accounting for the transport of the MF to the downstream region of the FS, it is clear that the radial and the tangential components are not equal. We therefore derive the plane-of-sky component of the MF as

$$
B_{\perp}=\sqrt{B_{t}^{2} \sin ^{2} \xi \cos ^{2} \alpha+B_{t}^{2} \cos ^{2} \xi+B_{r}^{2} \sin ^{2} \alpha+B_{t} B_{r} \sin \xi \sin (2 \alpha)},
$$

where $B_{r}$ is the radial component of the MF, $B_{t}=\sqrt{B_{\theta}^{2}+B_{\phi}^{2}}$ is the tangential component of the MF, $\alpha$ is the angle between the radial direction and the LOS, and $\xi$ is the angle between the 

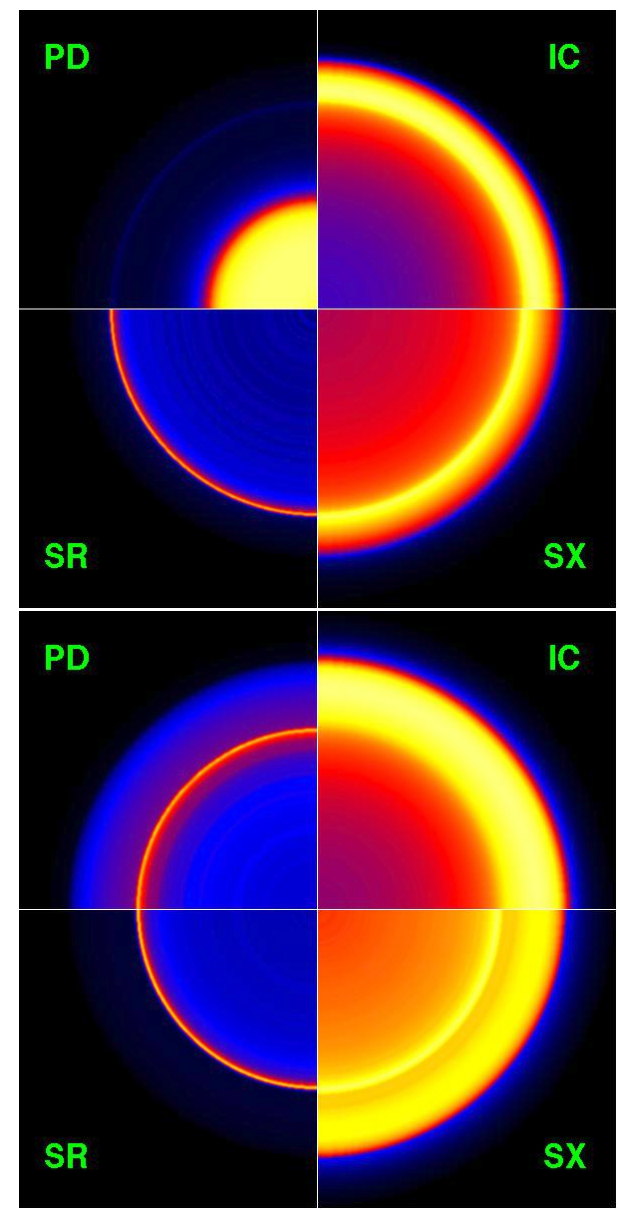
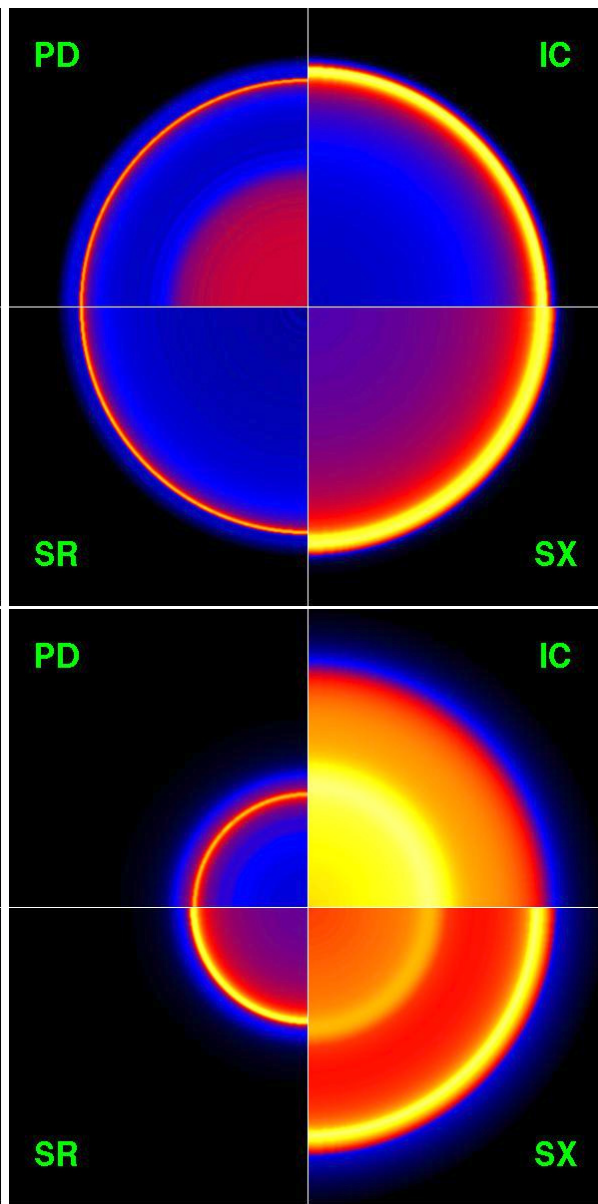

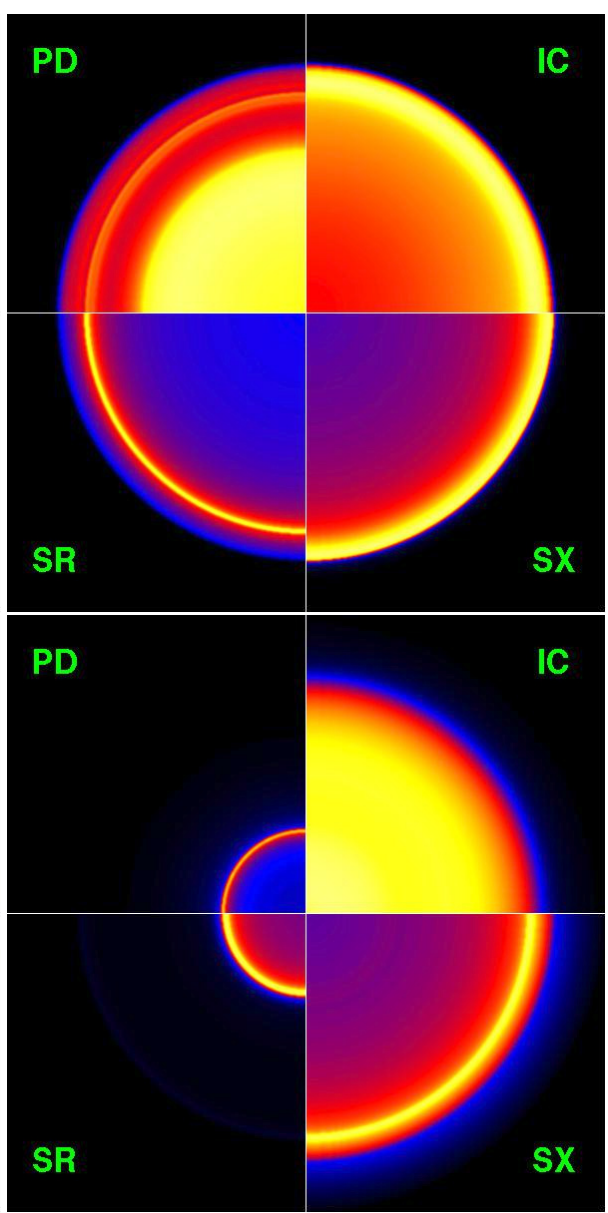

Fig. 7. Time evolution of intensity maps of type-Ic (top) and type-IIP (bottom) SNRs at $1 \mathrm{TeV}$ due to pion-decay (PD) and inverse Compton (IC), at $3 \mathrm{keV}(\mathrm{SX})$ and at $1.4 \mathrm{GHz}$ (SR) due to synchrotron radiation. The left column is for the age of 400 years, the middle column at 1000 years, and the right column at 1975 years. The scale is linear from zero to maximum in each image. All images are normalized by the FS radius, $R_{\mathrm{FS}}$, at the respective time.

direction of the tangential field and the $z$-axis. Since $\xi$ is unknown and in general may assume any value in the range $[0,2 \pi]$, there are two options to choose it. One option is to keep it constant at $\xi=\pi / 4$, the other one is to randomly vary it. The latter case would assume strong turbulence and little radio polarization (Stroman \& Pohl 2009). Here we chose $\xi=\pi / 4$ and applied the standard formula for synchrotron emission as for instance used by Sturner et al. (1997).

The IC emission was calculated using the full Klein-Nishina cross section (Blumenthal \& Gould 1970) for relativistic electrons following Sturner et al. (1997). As the target photon field for the IC scattering we considered the microwave background only. We calculated hadronic gamma-ray emission according to the procedure described by Huang et al. (2007).

Thermal emission was not considered here. However, we estimated this given the SNR parameters under consideration. Thermal emission should be very bright for young type-IIP SNR on account of the high gas density in the RSG wind. However, at very early times type IIP SNe tend to show the lowest X-ray intensity of all SN types (see Fig. 3 of Dwarkadas \& Gruszko 2012). The reason may be that the high density also provides strong absorption.

Most of the 1-TeV IC and pion-decay emission is created by particles of roughly $20-\mathrm{TeV}$ energy, whereas the energy of electrons producing $1.4-\mathrm{GHz}$ and $3-\mathrm{keV}$ synchrotron maps depends on the MF, which varies with age, location within the SNR, and type of SNR. The general trend to be noted from Fig. 6 is that the high-energy emission from type-Ic SNRs is dominated by IC emission, with a non-negligible contribution from piondecay only at the early age of 400 years. Figure 6 shows that the high-energy emission of type-IIP SNR is mostly hadronic, with a non-negligible contribution from IC only at the late age of 1975 years. This is not surprising, because the very thick RSG wind provides a good target for hadronic CR interactions in typeIIP SNR, whereas type-Ic SNRs expand in the low-density wind of the WR star. While the high-energy flux from type-Ic SNRs is increasing with time, that from type-IIP SNRs is decreasing. Type-IIP SNRs are always brighter in gamma-rays than type-Ic remnants with the exception of late times when the fluxes are of the same order. The synchrotron emission of both SNR types shows the opposite trend. To be noted from Figs. 6, 7 is that the spectra and intensity maps have a few distinct particle populations and emitting zones. Thus complex spectra and morphology arise naturally, but evolve in time.

At the age of 400 years, the hadronic emission from type-Ic SNRs comes from two regions: the region of dense ejecta provides a high-energy bump in the spectrum, and the region around the contact discontinuity accounts for a low-energy bump. The energetic CRs accelerated at the FS penetrate deep into the SNR and illuminate the ejecta, while low-energy CRs are trapped around the RS and illuminate gas at the nearby contact discontinuity. The IC emission at this time comes mostly from highenergy electrons of the FS. The synchrotron emission extends to hard X-rays. It comes mostly from the region between the 
FS and the contact discontinuity, somewhat increasing in intensity toward the CD on account of increasing MF (Rosenau \& Frankenthal 1976; Lyutikov \& Pohl 2004). The radio emission is clearly dominated by the emission from the $\mathrm{CD}$, where both $\mathrm{MF}$ and low-energy electrons are in excess. We point out that the decelerating CD is subject to the Rayleigh-Taylor instability, which we cannot treat in our 1D calculations. Therefore, real brightness maps would show a considerably more patchy structure near the contact discontinuity. The idealized picture presented here should nonetheless trace the general trend of the enhanced emission near the CD of core-collapse SNRs.

The type-IIP SNR at the same age shows quite different spectra and morphology of emission. Hadronic emission comes from the entire remnant with a significant contribution from the CD. The PD spectrum is therefore very soft because the emission region is dominated by the low-energy CR population accelerated at the RS. It is worth noting that the turnover in the PD spectrum is around $10 \mathrm{GeV}$, a feature observed in other core-collapse remnants as well (e.g. Cas A Acciari et al. 2010; Abdo et al. 2010). The IC emission is produced in the region between the CD and the FS, but its intensity is low. The whole SNR is very bright in synchrotron X-rays because the MF is high throughout. This makes it possible to clearly distinguish the RS, the CD, and the FS. The radio emission, as in type-Ic case, is dominated by the CD region.

At the age of 1000 years, the gamma-ray emission from typeIc SNR is dominated by IC radiation. The spectrum of the emission is double-peaked: at low energies it is dominated by the particles recently accelerated in the shock collision, whereas the high-energy IC emission is produced by particles accelerated at the FS. The PD spectrum is of low intensity with a significant bump at low energies. The IC and PD morphology maps at $1 \mathrm{TeV}$ are dominated by high-energy CRs, and therefore no hint of emission from RS-accelerated particles is seen. The PD emission of FS-accelerated protons illuminating the dense ejecta is still visible. The $\mathrm{CD}$ is now much brighter than the ejecta region, because the target density at the $\mathrm{CD}$ is on the same order as in the ejecta, but the CR density is much higher. The region between the CD and the FS, where the CR density is high, is bright in IC emission. Since the MF in the shocked region became approximately uniform, X-ray synchrotron emission predominantly comes from the same region, thus repeating the IC morphology. Likewise, the synchrotron spectrum largely resembles that of IC emission, except for minor differences on account of $\mathrm{MF}$ radial profile. The radio emission predominantly arises at the $\mathrm{CD}$, where the MF is higher than at the RS itself.

The very high gas density and fairly uniform distribution of CRs in 1000-year old type-IIP SNR renders the PD process dominant in the emission spectrum. However, the emissivity is mostly localized in the ejecta region. The $\mathrm{CD}$ is very bright, and the abundance of low-energy protons there makes the PD spectrum soft. The IC emission originates mostly in the weakly magnetized ejecta and the region between the two contact discontinuities (one behind the reverse shock and the other behind the intermediate shock). Both CDs are very prominent in synchrotron X-rays. The radio synchrotron map is again dominated by the CD behind the reverse shock on account of the distribution of low-energy electrons.

At 1975 years of age, the high-energy radiation from type-Ic SNRs is strongly dominated by IC scattering, with a significant contribution coming from low-energy electrons at the RS and ejecta regions. Most of the high-energy IC emission comes from the shocked region, where the electron number density is high. The PD radiation is very dim and arises mostly in the ejecta, with some contribution from the shocked region and the CD. Synchrotron X-rays are emitted in the region between the FS and the $\mathrm{CD}$, where both the number density of high-energy electrons and the MF are high. The radio emission is very bright around the CD and the RS, where the density peak of low-energy electrons is located and the MF is high. Since the ejecta have a very low MF, the low-energy peak in the synchrotron spectra has nearly disappeared. This demonstrates how the radial profile of the MF can affect the total emission spectrum, and that a simple scaling of the synchrotron emission to IC does not work.

The brightness of a 1975-year old type-IIP SNR is still dominated by the pion-decay emission coming from the compact ejecta region and the bright $\mathrm{CD}$, but only up to energies of around $1 \mathrm{TeV}$. At higher energies IC emission takes over, which arises throughout the remnant out to the intermediate shock, predominantly tracing regions of low MF. In the region of strong $\mathrm{MF}$, between the intermediate and forward shock, we observe hard X-ray synchrotron emission. Radio emission is significant in the ejecta region only. One can see that not only the spectra of IC and synchrotron emission do not resemble each other, but also their morphology is strikingly different. Thus care must be exercised in interpreting the observations if one attempts a reconstruction of the high-energy morphology from radio and X-ray synchrotron maps of core-collapse SNRs (Petruk et al. 2009). A clear shell-type SNR in X-rays may appear as a compact structure in high-energy gamma-rays, which may be mistaken for a pulsar-wind nebula, thus confusing the identification of high-energy gamma-ray sources. Note that the very dense CD and ejecta have not expanded significantly, and the radius of the $\mathrm{CD}$ is only around 3 pc after 1975 years. Resolving this region is a challenge for the current $\mathrm{TeV}$-band telescopes, because the angular diameter would be only $0.17^{\circ}$ at a distance of $2 \mathrm{kpc}$.

\section{Conclusions}

Using realistic models for the circumstellar medium created by stellar mass-loss around massive stars, we have studied the evolution of the resulting SNR shock waves within this medium, and computed the very high-energy signatures from the same. Our calculations of the medium into which SNRs evolve took into account the changes in mass-loss rate as the progenitor star evolved through various stages. In this way, our calculations far more precisely identified the various structures in the surrounding medium than those of previous authors. For instance, Caprioli (2011) assumed a single model to describe the circumstellar medium around all massive stars, with an RSG wind region extending into a constant density WR region. Not only is such a single description unsuitable over the large parameter space of stellar masses, but the structure these authors have described is not consistent with observations for massive stars of any given initial mass.

Ptuskin et al. (2010) also attempted to model the cosmicray acceleration for four types of SNRs, including three corecollapse types and type-Ia SN. However, the environments these authors assumed were not as detailed as ours, and it does not appear that they incorporated the density transitions that characterize the circumstellar medium in our work. As we have shown, the effects of these transitions make a large difference to the particle spectra and the radiation signatures.

We advanced our model of particle acceleration and propagation in SNRs (Telezhinsky et al. 2012a,b) by adding realistic transport of the MF. Our calculations suggest that the MF at the reverse shock is sufficient to accelerate particles to $\mathrm{TeV}$ energies. 
Although the maximum energy of cosmic-rays at the reverse shock is lower than at the FS, their intensity is high on account of the high ejecta density in core-collapse SNRs. Thus the reverse shock contributes a significant fraction of the total CR intensity in SNRs at early times. The RS-accelerated particles soften the total emission spectra, in agreement with recent data.

Emission spectra from core-collapse SNRs are complex and reflect both the distribution of gas or MF and the spectral differences between the particle populations coming from the forward and reverse shock. High-energy emission from type-Ic SNR is dominated by leptonic processes, particularly so when the SNR is older. High-energy radiation from type-IIP SNRs is very soft and strongly dominated by pion-decay emission, except for emission at the highest energies from old SNR.

Both types of remnants show, in addition to a shell structure, a center-filled morphology of high-energy emission, because the dense and weakly magnetized ejecta in the interior permits high-energy particles to propagate and radiate there. The shell-type morphology of type-IIP SNR, however, may be difficult to resolve with the current generation of gamma-ray telescopes. We also found that the complex radial distribution of MF causes significant differences in the morphology of synchrotron and IC emission from core-collapse SNRs, and therefore a "re-mapping" of the spectra and morphology of the two types of leptonic emission is not advised.

The high-energy emission from type-Ic SNRs is roughly an order of magnitude fainter than that of type-IIP SNRs at early ages, but type-Ic SNRs are good targets for the current generation of telescopes at the age of a thousand years and later, whereas type-IIP SNRs lose brightness very rapidly.

Our work demonstrates that the hydrodynamics of SNRs and their interaction with the environment play a significant role in shaping particle and emission spectra as well as intensity maps. The emission from core-collapse SNRs is significantly different from that of type-Ia SNRs that evolve in a uniform environment (Telezhinsky et al. 2012a).

\section{Appendix}

To derive Eq. (4) we rewrite Eq. (2) in spherical coordinates and use the properties of the spherically symmetric flow profile with velocity, $\boldsymbol{u}: u_{r}=u_{r}, u_{\theta}=0, u_{\phi}=0$ and $\partial u_{r} / \partial \theta=0, \partial u_{r} / \partial \phi=0$. The MF, $\boldsymbol{B}$, has all three components, $B_{r}, B_{\theta}, B_{\phi}$, and $\nabla \cdot \boldsymbol{B}=0$, therefore

$$
\begin{aligned}
& \boldsymbol{u}(\nabla\cdot \boldsymbol{B})=0 \\
& \boldsymbol{B}(\nabla \cdot \boldsymbol{u})= \\
& \quad \boldsymbol{B}\left(\frac{1}{r^{2}} \frac{\partial\left(r^{2} u_{r}\right)}{\partial r}+\frac{1}{r \sin \theta} \frac{\partial}{\partial \theta}\left(u_{\theta} \sin \theta\right)+\frac{1}{r \sin \theta} \frac{\partial u_{\phi}}{\partial \phi}\right) \\
&=B_{r}\left(\frac{2 u_{r}}{r}+\frac{\partial u_{r}}{\partial r}\right) \hat{\boldsymbol{r}}+B_{\theta}\left(\frac{2 u_{r}}{r}+\frac{\partial u_{r}}{\partial r}\right) \hat{\boldsymbol{\theta}}+B_{\phi}\left(\frac{2 u_{r}}{r}+\frac{\partial u_{r}}{\partial r}\right) \hat{\boldsymbol{\phi}} \\
&(\boldsymbol{B} \cdot \nabla) \boldsymbol{u}=\left(B_{r} \frac{\partial u_{r}}{\partial r}+\frac{B_{\theta}}{r} \frac{\partial u_{r}}{\partial \theta}+\frac{B_{\phi}}{r \sin (\theta)} \frac{\partial u_{r}}{\partial \phi}-\frac{B_{\theta} u_{\theta}+B_{\phi} u_{\phi}}{r}\right) \hat{\boldsymbol{r}} \\
&+\left(B_{r} \frac{\partial u_{\theta}}{\partial r}+\frac{B_{\theta}}{r} \frac{\partial u_{\theta}}{\partial \theta}+\frac{B_{\phi}}{r \sin (\theta)} \frac{\partial u_{\theta}}{\partial \phi}+\frac{B_{\theta} u_{r}}{r}-\frac{B_{\phi} u_{\phi} \cot (\theta)}{r}\right) \hat{\boldsymbol{\theta}} \\
&+\left(B_{r} \frac{\partial u_{\phi}}{\partial r}+\frac{B_{\theta}}{r} \frac{\partial u_{\phi}}{\partial \theta}+\frac{B_{\phi}}{r \sin (\theta)} \frac{\partial u_{\phi}}{\partial \phi}+\frac{B_{\phi} u_{r}}{r}+\frac{B_{\phi} u_{\theta} \cot (\theta)}{r}\right) \hat{\boldsymbol{\phi}} \\
&= B_{r} \frac{\partial u_{r}}{\partial r} \hat{\boldsymbol{r}}+B_{\theta} \frac{u_{r}}{r} \hat{\boldsymbol{\theta}}+B_{\phi} \frac{u_{r}}{r} \hat{\boldsymbol{\phi}}
\end{aligned}
$$

$$
\begin{aligned}
(\boldsymbol{u} \cdot \nabla) \boldsymbol{B}=\left(u_{r} \frac{\partial B_{r}}{\partial r}+\frac{u_{\theta}}{r} \frac{\partial B_{r}}{\partial \theta}+\frac{u_{\phi}}{r \sin (\theta)} \frac{\partial B_{r}}{\partial \phi}-\frac{u_{\theta} B_{\theta}+u_{\phi} B_{\phi}}{r}\right) \hat{\boldsymbol{r}} \\
+\left(u_{r} \frac{\partial B_{\theta}}{\partial r}+\frac{u_{\theta}}{r} \frac{\partial B_{\theta}}{\partial \theta}+\frac{u_{\phi}}{r \sin (\theta)} \frac{\partial B_{\theta}}{\partial \phi}+\frac{u_{\theta} B_{r}}{r}-\frac{u_{\phi} B_{\phi} \cot (\theta)}{r}\right) \hat{\boldsymbol{\theta}} \\
+\left(u_{r} \frac{\partial B_{\phi}}{\partial r}+\frac{u_{\theta}}{r} \frac{\partial B_{\phi}}{\partial \theta}+\frac{u_{\phi}}{r \sin (\theta)} \frac{\partial B_{\phi}}{\partial \phi}+\frac{u_{\phi} B_{r}}{r}+\frac{u_{\phi} B_{\theta} \cot (\theta)}{r}\right) \hat{\boldsymbol{\phi}} \\
=u_{r} \frac{\partial B_{r}}{\partial r} \hat{\boldsymbol{r}}+u_{r} \frac{\partial B_{\theta}}{\partial r} \hat{\boldsymbol{\theta}}+u_{r} \frac{\partial B_{\phi}}{\partial r} \hat{\boldsymbol{\phi}} .
\end{aligned}
$$

Combining the expressions, we find for the evolution of the three components of $\boldsymbol{B}$

$$
\begin{aligned}
\frac{\partial B_{r}}{\partial t} & =-2 B_{r} \frac{u_{r}}{r}-B_{r} \frac{\partial u_{r}}{\partial r}+B_{r} \frac{\partial u_{r}}{\partial r}-u_{r} \frac{\partial B_{r}}{\partial r} \\
& =-\frac{\partial}{\partial r}\left(B_{r} u_{r}\right)-2 B_{r} \frac{u_{r}}{r}+B_{r} \frac{\partial u_{r}}{\partial r}, \\
\frac{\partial B_{\theta}}{\partial t} & =-2 B_{\theta} \frac{u_{r}}{r}-B_{\theta} \frac{\partial u_{r}}{\partial r}+B_{\theta} \frac{u_{r}}{r}-u_{r} \frac{\partial B_{\theta}}{\partial r} \\
& =-\frac{\partial}{\partial r}\left(B_{\theta} u_{r}\right)-B_{\theta} \frac{u_{r}}{r}, \\
\frac{\partial B_{\phi}}{\partial t} & =-2 B_{\phi} \frac{u_{r}}{r}-B_{\phi} \frac{\partial u_{r}}{\partial r}+B_{\phi} \frac{u_{r}}{r}-u_{r} \frac{\partial B_{\phi}}{\partial r} \\
& =-\frac{\partial}{\partial r}\left(B_{\phi} u_{r}\right)-B_{\phi} \frac{u_{r}}{r} .
\end{aligned}
$$

Acknowledgements. We acknowledge support by the "Helmholtz Alliance for Astroparticle Physics HAP" funded by the Initiative and Networking Fund of the Helmholtz Association. V.V.D.'s work is supported by NASA Fermi grant NNX12A057G.

\section{References}

Abdo, A. A., Ackermann, M., Ajello, M., et al. 2010, ApJ, 710, L92

Acciari, V. A., Aliu, E., Arlen, T., et al. 2010, ApJ, 714, 163

Acciari, V. A., Aliu, E., Arlen, T., et al. 2011, ApJ, 730, L20

Acero, F., Aharonian, F., Akhperjanian, A. G., et al. 2010, A\&A, 516, A62

Aharonian, F., Akhperjanian, A. G., Bazer-Bachi, A. R., et al. 2007, ApJ, 661, 236

Aharonian, F., Akhperjanian, A. G., de Almeida, U. B., et al. 2009, ApJ, 692, 1500

Akiyama, S., Wheeler, J. C., Meier, D. L., \& Lichtenstadt, I. 2003, ApJ, 584, 954

Araya, M., \& Cui, W. 2010, ApJ, 720, 20

Atoyan, A., \& Dermer, C. D. 2012, ApJ, 749, L26

Axford, W. I., Leer, E., \& Skadron, G. 1977, in International Cosmic Ray Conference, International Cosmic Ray Conference, 11, 132

Bell, A. R. 1978, MNRAS, 182, 147

Beresnyak, A., Jones, T. W., \& Lazarian, A. 2009, ApJ, 707, 1541

Blandford, R. D., \& Ostriker, J. P. 1978, ApJ, 221, L29

Blasi, P., Gabici, S., \& Vannoni, G. 2005, MNRAS, 361, 907

Blasi, P., Morlino, G., Bandiera, R., Amato, E., \& Caprioli, D. 2012, ApJ, 755, 121

Blumenthal, G. R., \& Gould, R. J. 1970, Rev. Mod. Phys., 42, 237

Caprioli, D. 2011, J. Cosmology Astropart. Phys., 5, 26

Caprioli, D. 2012, J. Cosmology Astropart. Phys., 7, 38

Caprioli, D., Blasi, P., \& Amato, E. 2009, MNRAS, 396, 2065

Chevalier, R. A. 1982, ApJ, 259, 302

Chevalier, R. A., \& Fransson, C. 1994, ApJ, 420, 268

Chevalier, R. A., \& Oishi, J. 2003, ApJ, 593, L23

Colella, P., \& Woodward, P. R. 1984, J. Comput. Phys., 54, 174

DeLaney, T., Koralesky, B., Rudnick, L., \& Dickel, J. R. 2002, ApJ, 580, 914

Dwarkadas, V. V. 2005, ApJ, 630, 892

Dwarkadas, V. V. 2007, ApJ, 667, 226

Dwarkadas, V. V. 2011, Mem. Soc. Astron. It., 82, 781

Dwarkadas, V. V., \& Gruszko, J. 2012, MNRAS, 419, 1515

Dwarkadas, V. V., \& Rosenberg, D. L. 2013, High Energy Density Physics, 9, 226

Dwarkadas, V. V., Telezhinsky, I., \& Pohl, M. 2012, AIP Conf. Proc., 1505, 245 
Ellison, D. C., \& Bykov, A. M. 2011, ApJ, 731, 87

Ellison, D. C., Decourchelle, A., \& Ballet, J. 2005, A\&A, 429, 569

Ellison, D. C., Slane, P., Patnaude, D. J., \& Bykov, A. M. 2012, ApJ, 744, 39

Endeve, E., Cardall, C. Y., Budiardja, R. D., \& Mezzacappa, A. 2010, ApJ, 713, 1219

Endeve, E., Cardall, C. Y., Budiardja, R. D., et al. 2012, ApJ, 751, 26

Fransson, C., \& Björnsson, C.-I. 1998, ApJ, 509, 861

Garcia-Segura, G., Langer, N., \& Mac Low, M.-M. 1996, A\&A, 316, 133

Giacalone, J., \& Jokipii, J. R. 2007, ApJ, 663, L41

Giordano, F., Naumann-Godo, M., Ballet, J., et al. 2012, ApJ, 744, L2

Gotthelf, E. V., Koralesky, B., Rudnick, L., et al. 2001, ApJ, 552, L39

Guilet, J., Foglizzo, T., \& Fromang, S. 2011, ApJ, 729, 71

Guo, F., Li, S., Li, H., et al. 2012, ApJ, 747, 98

Helder, E. A., \& Vink, J. 2008, ApJ, 686, 1094

Huang, C., Park, S., Pohl, M., \& Daniels, C. D. 2007, Astropart. Phys., 27, 429

Inoue, T., Yamazaki, R., \& Inutsuka, S.-I. 2010, ApJ, 723, L108

Krymskii, G. F. 1977, Akademiia Nauk SSSR Doklady, 234, 1306

Lemoine-Goumard, M., Renaud, M., Vink, J., et al. 2012, A\&A, 545, A28

Lerche, I., Pohl, M., \& Schlickeiser, R. 2000, J. Plasma Phys., 64, 459

Lucek, S. G., \& Bell, A. R. 2000, MNRAS, 314, 65

Luo, Q., \& Melrose, D. 2009, MNRAS, 397, 1402

Lyutikov, M., \& Pohl, M. 2004, ApJ, 609, 785

Malkov, M. A., \& O'C Drury, L. 2001, Rep. Prog. Phys., 64, 429
Malkov, M. A., Diamond, P. H., \& Sagdeev, R. Z. 2012, Phys. Plasmas, 19, 082901

Martí-Vidal, I., Marcaide, J. M., Alberdi, A., et al. 2011, A\&A, 526, A143

Obergaulinger, M., \& Janka, H.-T. 2011 [arXiv: 1101.1198]

O'C. Drury, L., \& Downes, T. P. 2012, MNRAS, 427, 2308

Petruk, O., Bocchino, F., Miceli, M., et al. 2009, MNRAS, 399, 157

Ptuskin, V., Zirakashvili, V., \& Seo, E. 2010, ApJ, 718, 31

Rho, J., Dyer, K. K., Borkowski, K. J., \& Reynolds, S. P. 2002, ApJ, 581, 1116

Rosenau, P., \& Frankenthal, S. 1976, Phys. Fluids, 19, 1889

Sano, T., Nishihara, K., Matsuoka, C., \& Inoue, T. 2012, ApJ, 758, 126

Sasaki, M., Gaetz, T. J., Blair, W. P., et al. 2006, ApJ, 642, 260

Schure, K. M., Vink, J., Achterberg, A., \& Keppens, R. 2009, Adv. Space Res., 44,433

Stroman, W., \& Pohl, M. 2009, ApJ, 696, 1864

Sturner, S. J., Skibo, J. G., Dermer, C. D., \& Mattox, J. R. 1997, ApJ, 490, 619

Telezhinsky, I., Dwarkadas, V., \& Pohl, M. 2012a, Astropart. Phys., 35, 300

Telezhinsky, I., Dwarkadas, V. V., \& Pohl, M. 2012b, A\&A, 541, A153

Thompson, C., \& Duncan, R. C. 1993, ApJ, 408, 194

Toalá, J. A., \& Arthur, S. J. 2011, ApJ, 737, 100

van Marle, A. J., Langer, N., \& García-Segura, G. 2005, A\&A, 444, 837

Weaver, R., McCray, R., Castor, J., Shapiro, P., \& Moore, R. 1977, ApJ, 218, 377

Zirakashvili, V. N., \& Aharonian, F. A. 2010, ApJ, 708, 965

Zirakashvili, V. N., \& Ptuskin, V. S. 2012, Astropart. Phys., 39, 12 